\title{
The Mechanism Causing High-signal Intensity on Diffusion-weighted Imaging in Adnexal Torsion: Two Case Reports
}

\author{
Shinya Fujii ${ }^{1^{*}}$, Naoko Mukuda ${ }^{1}$, Kanae Nosaka ${ }^{2}$, Takeru Fukunaga ${ }^{1}$, \\ Chie Inoue $^{1}$, and Toshihide Ogawa ${ }^{1}$
}

\begin{abstract}
We report two cases with adnexal torsion to assess the mechanism of very strong signal intensity in ovarian lesions on DW imaging using MR imaging such as $\mathrm{T}_{2}^{*}$-weighted imaging. MR imaging findings of the cases suggest that the very strong signal intensity on DW imaging is contributed to cytotoxic edema with or without vasogenic edema, as well as oxyhemoglobin or extracellular methemoglobin.
\end{abstract}

Keywords: magnetic resonance imaging, diffusion-weighted magnetic resonance imaging, $T_{2}^{*}$-weighted imaging, ovary, ovarian

\section{Introduction}

Although ultrasonography is the first selected imaging modality for adnexal torsion, computed tomography (CT) and magnetic resonance (MR) imaging also play important roles for the diagnosis. ${ }^{1}$ Diffusion-weighted (DW) imaging has become accepted in body imaging for the detection and characterization of focal lesions. Many studies have reported the use of DW imaging in gynecological disease. In adnexal torsion, DW imaging shows strong signal intensity in the thickened fallopian tube and in the wall of cystic ovarian lesions. ${ }^{2}$ These findings can help to diagnose adnexal torsion and are suggested to reflect infarcted tissues and blood clots..$^{2-4} \mathrm{~A}$ recent report has shown that higher signal intensity, which is compared to that of nerve root on DW imaging, in the wall of ovarian tumor is significantly correlated with hemorrhagic infarction. ${ }^{4}$ However, the mechanism of the very strong signal intensity has not been resolved sufficiently. Besides, the pathological findings of torsed ovary are changed by the time from the onset. ${ }^{5}$ Therefore, the imaging findings accurately can reflect the pathological status at the time that MR imaging was preformed.

$\mathrm{T}_{2}^{*}$-weighted imaging $\left(\mathrm{T}_{2}^{*} \mathrm{WI}\right)$ has been widely accepted as a useful sequence for the identification of hemorrhage by demonstrating deoxyhemoglobin, intracellular methemoglobin and hemosiderin contents, particularly in neuroimaging. To our knowledge, there is only one report about ectopic

'Division of Radiology, Department of Pathophysiological and Therapeutic Science, Faculty of Medicine, Tottori University, 36-1 Nishi-cho, Yonago, Tottori 6838504, Japan

${ }^{2}$ Division of Organ Pathology, Department of Pathology, Faculty of Medicine, Tottori University, Tottori, Japan

*Corresponding author, E-mail: sfujii@grape.med.tottori-u.ac.jp

(O2016 Japanese Society for Magnetic Resonance in Medicine

This work is licensed under a Creative Commons Attribution-NonCommercialNoDerivatives International License.

Received: May 9, 2016 | Accepted: October 26, 2016 pregnancy describing the efficacy of $\mathrm{T}_{2}^{*} \mathrm{WI}$ applied for gynecological imaging. ${ }^{6}$ Herein, we demonstrate two cases with adnexal torsion, and assess the mechanism of very strong signal intensity in ovarian lesions on DW imaging using MR imaging such as $\mathrm{T}_{2}^{*} \mathrm{WI}$.

\section{Case Reports}

\section{Case 1}

48-year-old woman was referred to our hospital with acute lower abdominal pain. Serum biochemistry revealed increased white blood cell count $\left(10.8 \times 10^{3} / \mu \mathrm{L}\right)$ and CA19-9 $(98.2 \mathrm{U} / \mathrm{mL})$. A transvaginal ultrasonography (US) showed a multi-cystic mass in the pelvis. MR imaging was performed for further examination. The pelvic multi-cystic lesion demonstrated stained glass appearance with a twisted pedicle, which was compatible with adnexal torsion. The right-sided wall showed very strong signal intensity on DW imaging and hypo to mild hyperintensity on apparent diffusion coefficient (ADC) map. Besides, the wall showed isointensity on fatsuppressed $\mathrm{T}_{1}$-weighted imaging $\left(\mathrm{T}_{1} \mathrm{WI}\right)$, hyperintensity with marginal hypointensity on $\mathrm{T}_{2} \mathrm{WI}$ and $\mathrm{T}_{2}^{*} \mathrm{WI}$, and mild enhancement on subtraction dynamic contrast enhanced (DCE) imaging (Fig. 1). The patient underwent right salpingooophorectomy. At surgery, the pedicle was found to have a torsion of $540^{\circ}$ in a clockwise direction. Microscopic examination revealed mucinous cystic borderline tumor with hemorrhagic infarction. Diffuse or scattered hemorrhage was found particularly in inner and outer layer of the wall (Fig. 1).

\section{Case 2}

67-year-old woman was referred to our hospital with intermittent right lower abdominal pain of 1 month's duration. Her laboratory data was slightly elevated serum C-reactive protein $(0.31 \mathrm{mg} / \mathrm{dl})$ and increased CA125 $(56.4 \mathrm{U} / \mathrm{mL})$. A transvaginal US showed a unilocular cystic lesion. MR imaging was 


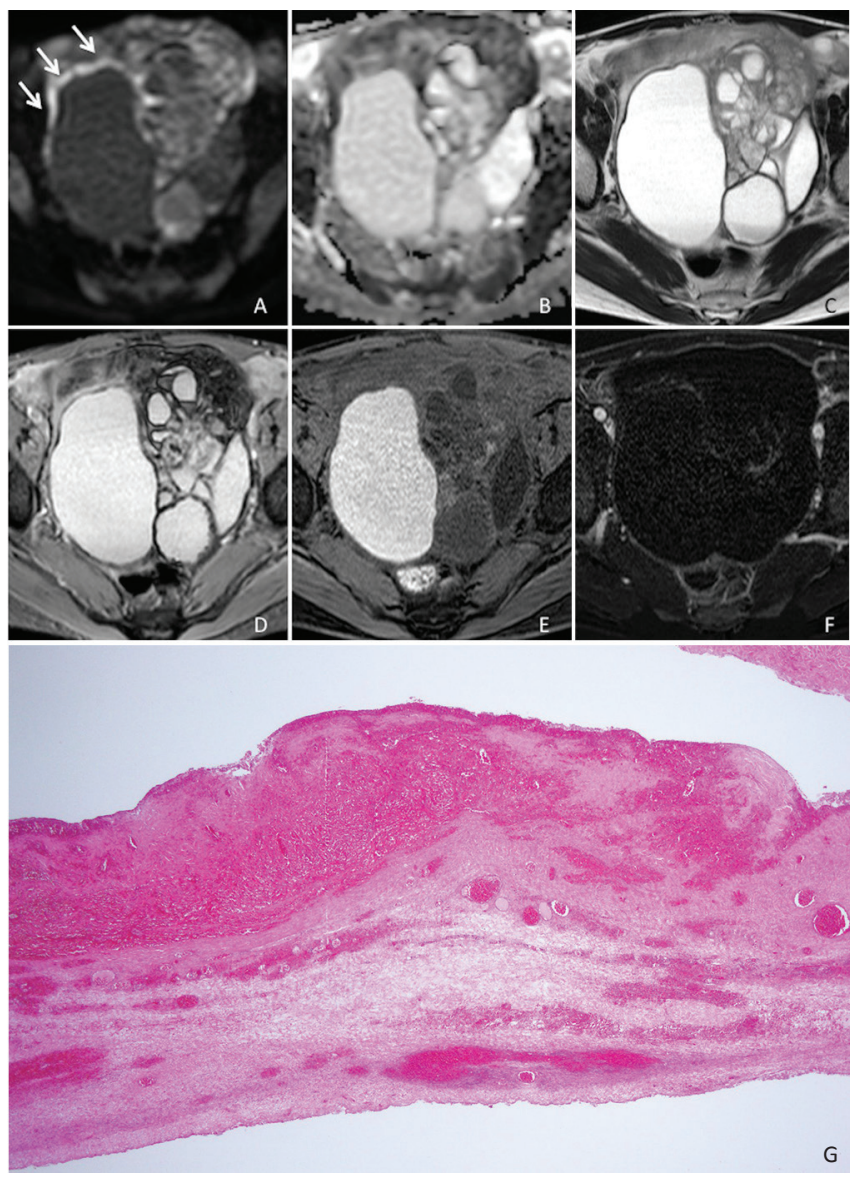

Fig 1. 48-year-old woman with twisted mucinous cystic borderline tumor in the right ovary. The right-sided wall of the mass shows very strong signal intensity on the diffusion-weighted (DW) image (A, arrows), and hypo to mild hyperintensity on the apparent diffusion coefficient (ADC) map (B). The wall shows isointensity on the fat-suppressed $\mathrm{T}_{1}$-weighted image (WI) (E) and hyperintensity with marginal hypointensity on the $\mathrm{T}_{2} \mathrm{WI}(\mathbf{C})$ and $\mathrm{T}_{2}^{*} \mathrm{WI}(\mathbf{D})$, and mild enhancement on the subtraction dynamic contrast enhanced (DCE) image (F). Diffuse or scattered hemorrhage is found particularly in inner and outer layer of the wall on hematoxylin-eosin stain with low-power field $(\mathbf{G})$.

performed for further examination. The right-sided wall of the mass showed very strong signal intensity on DW imaging and hypo to mild hyperintensity on ADC map. Besides, the wall showed hyperintensity on fat-suppressed $\mathrm{T}_{1} \mathrm{WI}$, hyperintensity with marginal hypointensity on $\mathrm{T}_{2} \mathrm{WI}$ and $\mathrm{T}_{2}^{*} \mathrm{WI}$, and mild enhancement on subtraction DCE imaging (Fig. 2). The patient underwent right salpingo-oophorectomy. At surgery, the pedicle was found to have a torsion of $1080^{\circ}$ in a clockwise direction. Microscopic examination revealed serous cystadenoma with hemorrhagic infarction. Hemosiderin depositions were scattered in the cyst wall (Fig. 2).

\section{Discussion}

The area with very strong signal intensity on DW imaging showed hypointensity or mild hyperintensity on the ADC
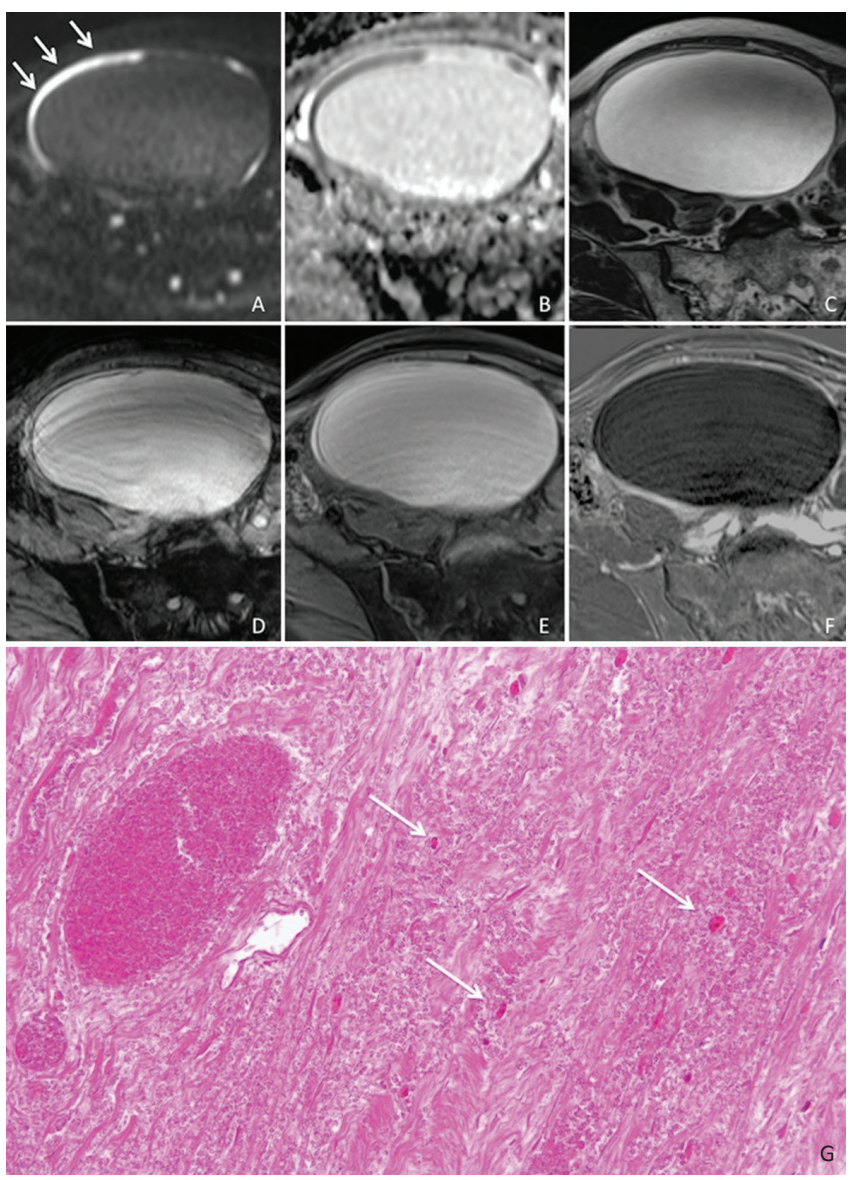

Fig 2. 67-year-old woman with twisted serous cystadenoma in the right ovary. The right-sided wall of the mass shows very strong signal intensity on the diffusion-weighted (DW) image (A, arrows), and hypo to mild hyperintensity on the apparent diffusion coefficient (ADC) map (B). The wall shows hyperintensity on the fat-suppressed $\mathrm{T}_{1}$-weighted image (WI) (E) and hyperintensity with marginal hypointensity on the $T_{2} W I(C)$ and $\mathrm{T}_{2}^{*} \mathrm{WI}(\mathbf{D})$, and mild enhancement on the subtraction dynamic contrast enhanced (DCE) image $(\mathbf{F})$. Hemosiderin depositions are scattered in the cyst wall on hematoxylin-eosin stain with high-power field (G, arrows).

maps in present cases. According to the report about cerebral venous infarction, the lesion shows a decreased ADC reflecting cytotoxic edema induced by tissue hypoperfusion. Over time, the ADC increases because of resolving cytotoxic edema and subsequent increasing vasogenic edema. ${ }^{7}$ Therefore, the findings on the ADC maps are considered to reflect cytotoxic edema with or without vasogenic edema.

The areas showed isointensity or hyperintensity on fatsuppressed $\mathrm{T}_{1} \mathrm{WI}$, and hyperintensity with marginal hypointensity on $\mathrm{T}_{2} \mathrm{WI}$ and $\mathrm{T}_{2}^{*} \mathrm{WI}$. We speculate that the imaging findings reflect not only the aforementioned edema but also hemorrhage. Hypointensity on $\mathrm{T}_{2}^{*} \mathrm{WI}$ demonstrates hemorrhage reflecting deoxyhemoglobin, intracellular methemoglobin, and hemosiderin contents. In case1 with acute onset, we suggest that the marginal hypointensity reflects deoxyhemoglobin, while the central area may reflect 
oxyhemoglobin. On the other hand, in case 2 with gradual onset, the marginal hypointensity on $\mathrm{T}_{2}$ and $\mathrm{T}_{2}^{*} \mathrm{WI}$ may reflect hemosiderin while the central area may reflect extracellular methemoglobin showing hyperintensity on both $\mathrm{T}_{2} \mathrm{WI}$ and fat-suppressed $\mathrm{T}_{1} \mathrm{WI}$. Additionally, $\mathrm{T}_{2}$-hyperintense hematomas show hyperintense on DW images due to $\mathrm{T}_{2}$ shine-through effect. ${ }^{8}$ Therefore, hemorrhage is suggested to contribute to hyperintensity on DW imaging.

Meanwhile, the areas showed mild enhancement on DCE imaging. Adnexal torsion produces circulatory stasis that is initially venous, but becomes arterial with progression of the torsion and resultant edema, followed by the obstruction of the arterial blood supply. ${ }^{1}$ The walls with mild enhancement are considered to indicate the obstructed venous flow with arterial blood supply.

In gynecological imaging, reports concerning the identification of hemorrhage using $\mathrm{T}_{2}^{*} \mathrm{WI}^{6}$ and susceptibilityweighted imaging (SWI) have recently increased. ${ }^{9}$ Regarding ovarian torsion, ${ }^{9}$ the case of subacute torsion showed prominent signal voids in the twisted vascular pedicle and peripheral region of fibrothecoma on SWI. In general, SWI is more sensitive in detecting the foci than $\mathrm{T}_{2}^{*} \mathrm{WI}$. However, $\mathrm{T}_{2}^{*} \mathrm{WI}$ is more feasible than SWI because of less time-consuming method; the acquisition time of $\mathrm{T}_{2}^{*} \mathrm{WI}$ is only $60 \mathrm{sec}$. Therefore, we consider that $\mathrm{T}_{2}^{*} \mathrm{WI}$ is more feasible in clinical routine practice. However, $\mathrm{T}_{2}^{*} \mathrm{WI}$ cannot differentiate blood products from calcification without unenhanced CT although SWI phase images can be used to distinguish calcification (low-signal intensity) from hemorrhagic regions (high-signal intensity). ${ }^{10}$

Hemorrhage on the surface of cyst wall may occur in tubo-ovarian abscesses and endometriotic cysts. However, in tubo-ovarian abscesses, not the wall but the content fluid shows hyperintensity reflecting viscosity on DW imaging. Meanwhile, endometriotic cysts generally show hyperintensity similar to fat on $\mathrm{T}_{1} \mathrm{WI}$ and shading on $\mathrm{T}_{2} \mathrm{WI}$, which are characteristic imaging findings. Therefore, we can differentiate adnexal torsion from these pathological conditions.

In conclusion, in adnexal torsion, very strong signal intensity on DW imaging is contributed to cytotoxic edema with or without vasogenic edema, as well as oxyhemoglobin or extracellular methemoglobin. Besides, the same area showing two layers on $\mathrm{T}_{2}$ and $\mathrm{T}_{2}^{*} \mathrm{WI}$ possibly reflected deoxyhemoglobin and oxyhemoglobin in case 1 with acute onset, hemosiderin and extracellular methemoglobin in case 2 with gradual onset.

\section{Conflicts of Interest}

The authors declare that they have no conflicts of interest.

\section{References}

1. Kimura I, Togashi K, Kawakami S, Takakura K, Mori T, Konishi J. Ovarian torsion: CT and MR imaging appearances. Radiology 1994; 190:337-341.

2. Fujii S, Kaneda S, Kakite S, et al. Diffusion-weighted imaging findings of adnexal torsion: initial results. Eur J Radiol 2011; 77:330-334.

3. Kato H, Kanematsu M, Uchiyama M, Yano R, Furui T, Morishige K. Diffusion-weighted imaging of ovarian torsion: usefulness of apparent diffusion coefficient (ADC) values for the detection of hemorrhagic infarction. Magn Reson Med Sci 2014; 13:39-44.

4. Moribata Y, Kido A, Yamaoka T, et al. MR imaging findings of ovarian torsion correlate with pathological hemorrhagic infarction. J Obstet Gynaecol Res 2015; 41:1433-1439.

5. Taskin O, Birincioglu M, Aydin A, et al. The effects of twisted ischaemic adnexa managed by detorsion on ovarian viability and histology: an ischaemia-reperfusion rodent model. Hum Reprod 1998; 13:2823-2827.

6. Yoshigi J, Yashiro N, Kinoshita T, O'uchi T, Kitagaki H. Diagnosis of ectopic pregnancy with MRI: efficacy of $\mathrm{T}_{2}^{*}$-weighted imaging. Magn Reson Med Sci 2006; 5:25-32.

7. Forbes KP, Pipe JG, Heiserman JE. Evidence for cytotoxic edema in the pathogenesis of cerebral venous infarction. AJNR Am J Neuroradiol 2001; 22:450-455.

8. Silvera S, Oppenheim C, Touzé E, et al. Spontaneous intracerebral hematoma on diffusion-weighted images: influence of $\mathrm{T}_{2}$-shine-through and $\mathrm{T}_{2}$-blackout effects. AJNR Am J Neuroradiol 2005; 26:236-241.

9. Takeuchi M, Matsuzaki K, Harada M. Susceptibilityweighted Imaging of Ovarian Torsion: A Case Report. Magn Reson Med Sci 2015; 14:355-358.

10. Löbel U, Sedlacik J, Sabin ND, et al. Three-dimensional susceptibility-weighted imaging and two-dimensional $\mathrm{T}_{2}^{*}$-weighted gradient-echo imaging of intratumoral hemorrhages in pediatric diffuse intrinsic pontine glioma. Neuroradiology 2010; 52:1167-1177. 